\title{
Plasma convection jets near the poleward boundary of the nightside auroral oval and their relation to Pedersen conductivity gradients
}

\author{
H. Wang ${ }^{1, *}$, H. Lühr ${ }^{2}$, and A. J. Ridley ${ }^{3}$ \\ ${ }^{1}$ Dept. of Space Physics, School of Electronic Information, Wuhan University, Wuhan 430079, China \\ ${ }^{2}$ Helmholtz Centre Potsdam-GFZ, German Research Center for Geosciences, 14473 Potsdam, Germany \\ ${ }^{3}$ Department of Atmospheric, Oceanic, and Space Sciences, University of Michigan, Ann Arbor, MI-48109, USA \\ *also at: State Key Laboratory of Space Weather, Chinese Academy of Sciences, Beijing 100080, China
}

Received: 29 October 2009 - Revised: 19 March 2010 - Accepted: 12 April 2010 - Published: 15 April 2010

\begin{abstract}
In this work, we have shown that the ionospheric azimuthal plasma velocity jets near the open-closed field line boundary on the nightside can be associated with the peak in the ionospheric conductivity gradient. Both model and DMSP observations have been utilized to conduct this investigation. The model tests show that when the gradient of conductivity in the poleward boundary becomes sharper, convection peaks appear around the poleward edge of the aurora. The model results have been confirmed by DMSP observations. Hundreds of large ion flow events are identified from one year DMSP observations, with flow speed larger than $500 \mathrm{~m} / \mathrm{s}$ that occurred poleward of the aurora. Among them, $280(74 \%)$ events are found to be associated with conductivity gradient peaks. Most of the convection jets occur in winter when conductivity gradients are expected to be large. The convection jets tend to occur at later local times (21:0022:00 MLT) at $70^{\circ}-72^{\circ}$ MLat. These events are preceded by increasing of the merging electric field suggesting that they occur after the expansion of the polar cap. Both observation and model results show that the conductivity gradient at the polar cap boundary are one of the important elements in establishing the convection jets.
\end{abstract}

Keywords. Ionosphere (Electric fields and currents; Plasma convection) - Magnetospheric physics (Polar cap phenomena)

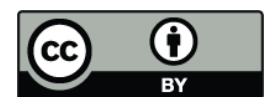

Correspondence to: $\mathrm{H}$. Wang

(h.wang@whu.edu.cn)

\section{Introduction}

Electric fields from the solar wind are mapped to the high latitude ionosphere, creating horizontal $\boldsymbol{E} \times \boldsymbol{B}$ plasma drift, also called convection. The convection depends on the IMF direction (e.g. Heppner and Maynard, 1987; Weimer, 1996). The convection pattern can be measured using satellites, radars, and ground based magnetometers (e.g Heppner and Maynard, 1987; Ruohoniemi and Greenwald, 1996; Richmond and Kamide, 1988; Lyons et al., 1996; Wang et al., 2008). When global patterns of the convection are compiled, they are typically smoothed and there are few localized features in the pattern. When individual traces of satellite orbits are examined, however, significant structure is often observed. For example, intense ionospheric F-region east-west flow jets or enhanced electric fields are frequently recorded by satellites at high latitude near the open-closed field line boundary (e.g. Karlsson and Marklund, 1996; Burke et al., 1994; Hamza et al., 2000; Ridley et al., 2002).

The rapid plasma flow jets on the dayside (06-12-18 MLT) have been studied widely in the literature, including their dependencies on solar wind, IMF conditions and seasons (e.g. Clauer and Ridley, 1995; Ridley and Clauer, 1996; Provan and Yeoman, 1999; Milan et al., 2001; Ruohoniemi and Greenwald, 2005). Several mechanisms were proposed for this phenomena, such as Kelvin-Helmholtz instability (e.g. Clauer and Ridley, 1995; Ridley and Clauer, 1996) or IMF $B_{\mathrm{y}}$ related reconnection (Provan and Yeoman, 1999). Furthermore, Milan et al. (2001) found that the influence of IMF $B_{\mathrm{y}}$ on the dayside flow bursts is greater in the summer than in the winter.

Opposed to the dayside, the rapid plasma flow jet on the nightside aurora boundary has been rarely studied statistically. Only event studies have been conducted, and it was suggested that the plasma flow burst events in the midnight

Published by Copernicus Publications on behalf of the European Geosciences Union. 
sector are related to the processes in the magnetotail plasma sheet boundary, which might be an element of the substorm (e.g. Burke et al., 1994; Hamza et al., 2000) or to the convective transport after reconnection in the more distant tail, not directly related to substorms (Grocott et al., 2004; Grocott et al., 2008). These studies have suggested the plasma fast flow is attributed mainly to magnetotail processes. Since the open-closed field line boundary is proximate to the poleward boundary of the auroral oval, it is worthwhile to investigate whether the flow bursts may be due to the conductivity structure.

Previous studies have shown that ionospheric conductivity has a strong effect on the magnetospheric dynamics (e.g. Raeder et al., 2001). Fedder and Lyon (1987) proposed that on a global scale, as Pedersen conductivity increases, the electric potential will decrease, and the current will change. This makes the magnetospheric dynamo to look like neither an intrinsic voltage source nor an intrinsic current source. Simplistically, one expects that when the conductivity decreases, the flow velocities will increase. Therefore, the fast flows observed by DMSP may be caused by a decrease in conductivity. Previous studies have shown that gradients in the Hall conductivity can cause the potential pattern to be asymmetric from dawn to dusk (e.g. Ridley et al., 2004; Sandholt and Farrugia, 2009). However, statistical studies are still failing to explicitly describe the effect of the ionospheric conductivity gradient on the nightside fast flows. This paper examines whether gradients in the Pedersen conductance plays a role in the development of fast flow jets.

Spatial and temporal variations of the ionospheric conductivity are primarily due to solar radiation and precipitation of magnetospheric particles. The particle precipitation-related Pedersen conductivity depends primarily on the electron average energy and energy flux (Hardy et al., 1987), while the solar radiation induced conductivity depends on both the level of extreme ultraviolet (EUV), which is approximated by the F10.7 index, and the solar zenith angle. The solar driven conductivity varies smoothly over large spatial scales, while the aurora can vary radically over small scales. It is therefore clear that if the gradient in the Pedersen conductance causes spatially confined fast flows to occur, it will most likely happen near the auroral oval.

In this paper, we investigate the dependence of the nightside convection jet on the conductivity gradient by using both model results and DMSP satellite observations.

\section{Model results}

The relationship between the electric potential $(\Phi)$ and the radial component of the field-aligned currents $\left(j_{\mathrm{R}}\right)$ is $j_{\mathrm{R}}=$ $\nabla_{\perp} \cdot(\Sigma \cdot \nabla \Phi)$, where $\Sigma$ is the ionospheric conductance tensor. This relationship can be expanded as follows (e.g. Good- man, 1995; Amm, 1996),

$$
\begin{aligned}
j_{\mathrm{R}}(R, \theta, \psi)= & \frac{1}{R^{2}}\left[\frac{\delta^{2} \Phi}{\delta \theta^{2}} \frac{\Sigma_{0} \Sigma_{\mathrm{P}}}{C}+\right. \\
& \frac{\delta^{2} \Phi}{\delta \psi^{2}}\left\{\frac{1}{\sin ^{2} \theta}\left(\Sigma_{\mathrm{P}}+\frac{\Sigma_{\mathrm{H}}^{2} \sin ^{2} \varepsilon}{C}\right)\right\}+ \\
& \frac{\delta \Phi}{\delta \theta}\left\{\frac{\delta}{\delta \theta}\left(\frac{\Sigma_{0} \Sigma_{\mathrm{P}}}{C}\right)+\cot \theta \frac{\Sigma_{0} \Sigma_{\mathrm{P}}}{C}+\right. \\
& \left.\frac{1}{\sin \theta} \frac{\delta}{\delta \psi}\left(\frac{\Sigma_{0} \Sigma_{\mathrm{H}} \cos \varepsilon}{C}\right)\right\}+ \\
& \frac{\delta \Phi}{\delta \psi}\left\{\frac{\delta}{\delta \theta}\left(\frac{\Sigma_{0} \Sigma_{\mathrm{H}}(-\cos \varepsilon)}{C \sin \theta}\right)+\right. \\
& \frac{1}{\sin ^{2} \theta} \frac{\delta}{\delta \psi}\left(\Sigma_{\mathrm{P}}+\frac{\Sigma_{\mathrm{H}}^{2} \sin ^{2} \varepsilon}{C}\right)+ \\
& \left.\left.\frac{\Sigma_{0} \Sigma_{\mathrm{H}}(-\cos \varepsilon) \cot \theta}{C \sin \theta}\right\}\right]
\end{aligned}
$$

Where $C=\Sigma_{0} \cos ^{2} \varepsilon+\Sigma_{\mathrm{P}} \sin ^{2} \varepsilon, \varepsilon$ is the angle between the radial direction and the magnetic field, $\theta$ is colatitude, $\psi$ is longitude, and $\Sigma_{0}, \Sigma_{\mathrm{P}}$, and $\Sigma_{\mathrm{H}}$ are field-aligned, Pedersen, and Hall height integrated conductances, respectively (for more information, the reader is referred to Ridley et al., 2004).

By assuming that the potential and conductivity are homogeneous in the longitudinal direction (this is common near dawn and dusk), and that the field lines are vertical, which is valid in the high latitude region, we can simplify Eq. (1) as

$j_{\mathrm{R}}=\frac{1}{R^{2}}\left(\frac{\delta \Sigma_{\mathrm{P}}}{\delta \theta} \frac{\delta \Phi}{\delta \theta}+\Sigma_{\mathrm{P}} \frac{\delta^{2} \Phi}{\delta^{2} \theta}\right)$

The northward directed electric field can then be described as $\boldsymbol{E}_{\theta}=-\frac{\delta \Phi}{\delta \theta}$. The above relationship can be expressed as $j_{\mathrm{R}}=-\frac{1}{R^{2}}\left(\frac{\delta}{\delta \theta}\left(\Sigma_{\mathrm{P}} \boldsymbol{E}_{\theta}\right)\right)$. This reflects a simple relationship between the northward directed electric field $\left(\boldsymbol{E}_{\theta}\right)$ and Pedersen conductance $\left(\Sigma_{\mathrm{P}}\right)$.

Solving the above function in a numerical way, we have assumed that duskside R1 and R2 FACs exhibit sin wave shaped latitudinal profiles in the auroral region, as shown in Fig. 1, while the auroral Pedersen conductance is shaped as a sin wave (left) and $\sin ^{0.25}$ wave (right) superposed on a $1 \mathrm{~S}$ background conductance. Using sin wave and $\sin ^{0.25}$ wave shaped conductance, we have run the model twice.

The left figure assumes that the auroral boundaries are quite smooth, while the right figure shows the aurora to have sharp boundaries, with strong gradients. The electric potential and electric field can then be solved by using Eq. (2). This solution is shown as solid line. We then determine the contribution from the gradient in the conductivity by neglecting the term $\frac{\delta \Sigma_{\mathrm{P}}}{\delta \theta}$ (i.e. setting it to zero) in Eq. (2). This results in the dotted curve.

It can be seen, that for $\frac{\delta \Sigma_{\mathrm{P}}}{\delta \theta}=0$ the electric field structure does not change much when the conductivity gradient becomes steeper at the edges, when comparing the left plot 
to the right. Since there is more conductivity in the $\sin ^{0.25}$ case, the electric field (when the conductivity gradient is neglected) is diminished. But for $\frac{\delta \Sigma_{P}}{\delta \theta} \neq 0$, when the gradient of conductivity becomes larger, the electric field structure becomes more complicated with several peaks occurring at the edges. Since the Pedersen conductivity on the nightside can change suddenly and dramatically at the boundary of the auroral oval, the $\frac{\delta \Sigma_{\mathbf{p}}}{\delta \theta}$ term is expected to have an important effect on the electric field structure. From this analysis, we predict that jumps in cross-track velocity are associated with the sudden changes in the ionospheric conductivity. The negative peaks in $V_{\mathrm{y}}$ (anti-sunward flow) near $75^{\circ}$ latitude might resemble flow bursts in DMSP observations.

\section{Satellite observations of fast flows}

The DMSP satellites sample polar regions at $\sim 835 \mathrm{~km}$ altitude along orbits of fixed local times. The orbital period is approximately $100 \mathrm{~min}$. One of the satellites (F13) has a near dawn-dusk orbit and two (F14, F15) have 09:3021:30 MLT orbits. The DMSP satellite tracks are confined to the MLT sector of 15:00-22:00 MLT, leaving a midnight gap in the MLT coverage. Among various scientific instruments onboard, the instruments of primary interest for this study are the ion drift meter (IDM) and the electron spectrometers (SSJ/4). The ion drift velocities in the horizontal and vertical direction perpendicular to the satellite track are derived from the IDM data (Rich and Hairston, 1994). The DMSP SSJ/4 instruments monitor the energy flux of electrons and ions in the range of $30 \mathrm{eV}$ to $30 \mathrm{keV}$ that precipitate from the Earth's magnetosphere (Hardy et al., 1984). Robinson et al. (1987) have described the relationship relating the energy flux and the average energy of the electrons with the height-integrated ionospheric conductivity. Here we make use of this empirical relation.

For this study, rapid convection flow is defined as a clearly identifiable large ion flow poleward of the aurora oval. A threshold of flow velocity greater than $500 \mathrm{~m} / \mathrm{s}$ is used for selection. The poleward boundary of the aurora oval is found automatically by computing the auroral Pedersen conductance along the DMSP path and determining the peak conductance, then stepping poleward until the conductance is reduced to 0.2 times the peak value or $1 \mathrm{~S}$, whichever is smaller. These criteria are based on the finding of Troshichev et al. (1996) that the diffuse electron and ion precipitation is one order of magnitude or more weaker in the polar cap than in the auroral oval. The selected orbits are further visually inspected to fully satisfy the above criteria. One year of DMSP measurements during 2002 have been processed and 378 rapid convection flow events are identified. Among them, $280(74 \%)$ events are associated with conductivity gradient peaks. These 280 events are considered and termed convection jet events in the following.
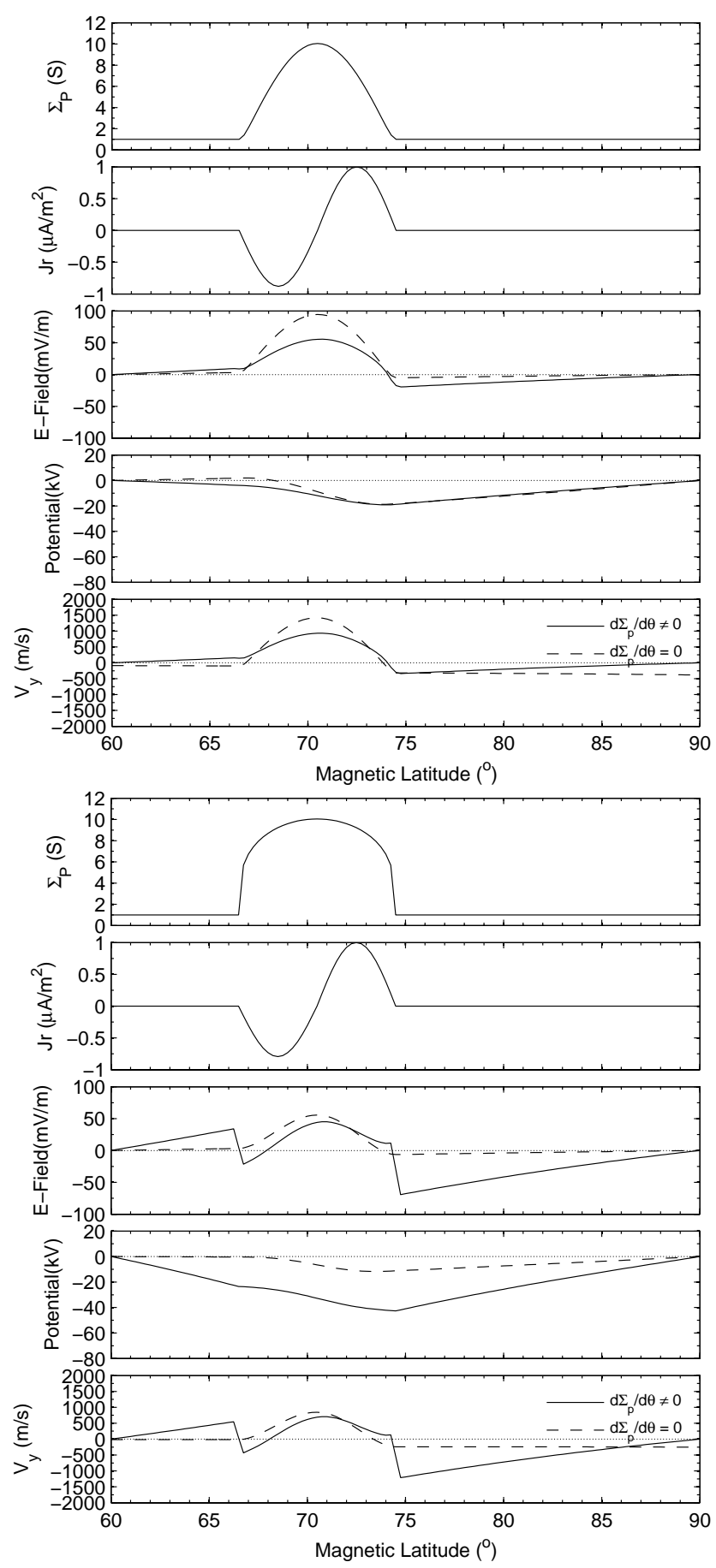

Fig. 1. Numerical simulation of high latitude plasma dynamics. From top to bottom frames show the Pedersen conductivity (sin wave on the top, $\sin ^{0.25}$ wave on the bottom), field-aligned currents (FACs, sin wave) and the derived electric field, potential, and azimuthal velocity (positive denotes sunward) from Eq. (2) (real line). Also shown are the derived electric field, potential, and azithual velocity when the conductivity gradient is neglected in Eq. (2) for comparison (dashed line). 

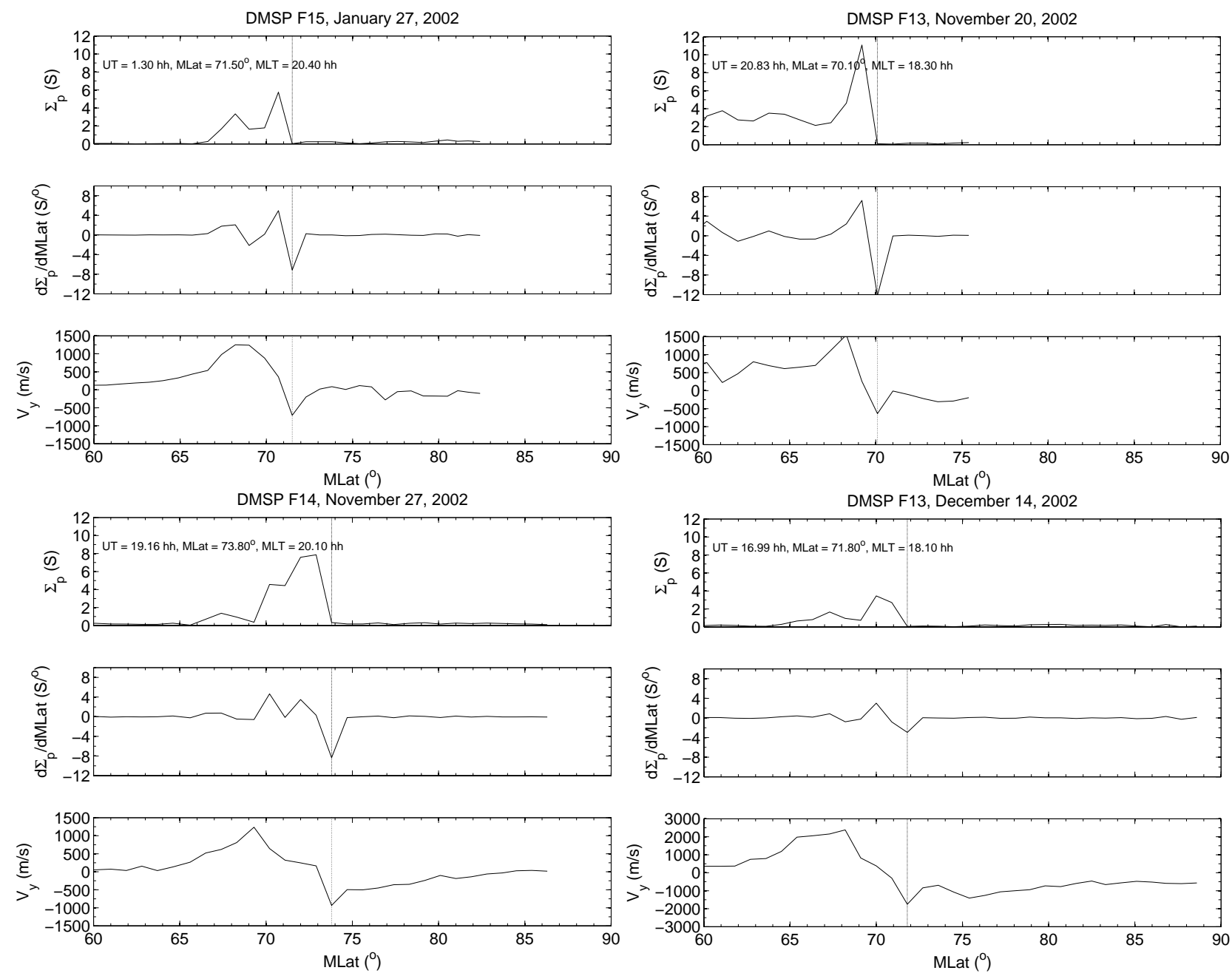

Fig. 2. Examples of the observed latitude profile of Pedersen conductivity $\left(\Sigma_{\mathrm{P}}\right)$, its latitudinal gradient $\left(d \Sigma_{\mathrm{p}} / d \mathrm{MLat}\right)$, and the ionospheric convection velocity $\left(V_{\mathrm{y}}\right)$ as obtained from DMSP F13 on 20 November 2002 and 14 December 2002, F14 on 27 November 2002 , and F15 on 27 January 2002. The enhanced anti-sunward (negative) plasma drift near the auroral boundary is the plasma jet, which is coincident with the jump of the Pedersen Conductivity. The peak velocity of the jet is indicated by the dashed line. The UT, MLat, and MLT where the velocity peak occurs are also given.

Four examples of convection jets in the north polar region are shown in Fig. 2. From top to bottom it shows latitude profiles of Pedersen conductance due to particle precipitation, the latitudinal gradient of the Pedersen conductance, and the ionospheric convection. The enhanced conductivity due to particle precipitation represents the auroral oval. The large sunward (positive) flow in this region is the auroral plasma convection. For all events the aurora has clear poleward boundaries, that is, with strong latitudinal gradients. The enhanced anti-sunward (negative) plasma drift poleward of the auroral precipitation is the convection jet. The UT, MLat (magnetic latitude), and MLT (magnetic local time) where the velocity peak occurs are listed in the plots.

The time (UT), position (MLT, MLat) and magnitude of peak velocities of the convection jets are listed in a catalog for each event. All these parameters describe the position where the jet velocity peaks. Only the convection jet observations in the Northern Hemisphere are considered in this study, and the inter-hemispheric comparison will be left for future studies.

\section{Statistical results}

\subsection{MLT and MLat distribution}

The identified jet events are binned by magnetic local time ( $1 \mathrm{~h}$ bins). The number of events has been normalized to the number of DMSP passes over that MLT bin. Figure 3 (left frame) shows the occurrence frequency of jet events 

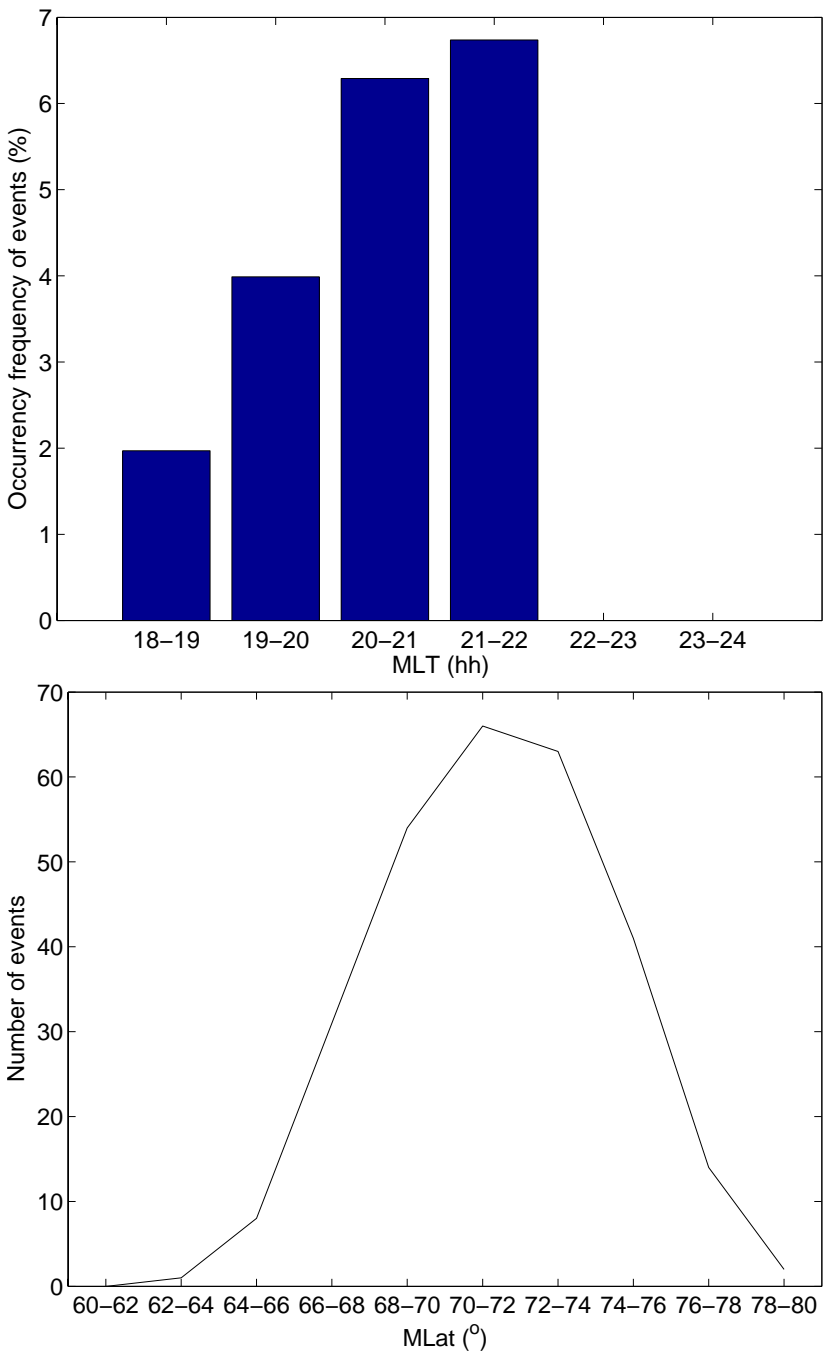

Fig. 3. Distribution of fast plasma jets. Top shows the occurrence frequency of convection jets as a function of MLT. Bottom shows the number of events as a function of MLat.

as a function of MLT. The bars reflect the percentage of DMSP passes that measure jets. Jets exhibit occurrence peaks around 21:00-22:00 MLT. We do not have data after 22:00 MLT so we do not know what happened to the distribution outside this MLT sector.

The jets are then binned by magnetic latitude ( $2^{\circ}$ bins). Figure 3 (right frame) shows the resulting distributions of the number of the jet events as a function of MLat, reflecting a clearly preferred MLat location. The distribution peaks at $70^{\circ}-72^{\circ}$ MLat.

\subsection{Solar wind and IMF condition}

It would be interesting to see whether there are interplanetary configurations favorable for the jet events to occur. The solar wind and interplanetary magnetic field (IMF) con-

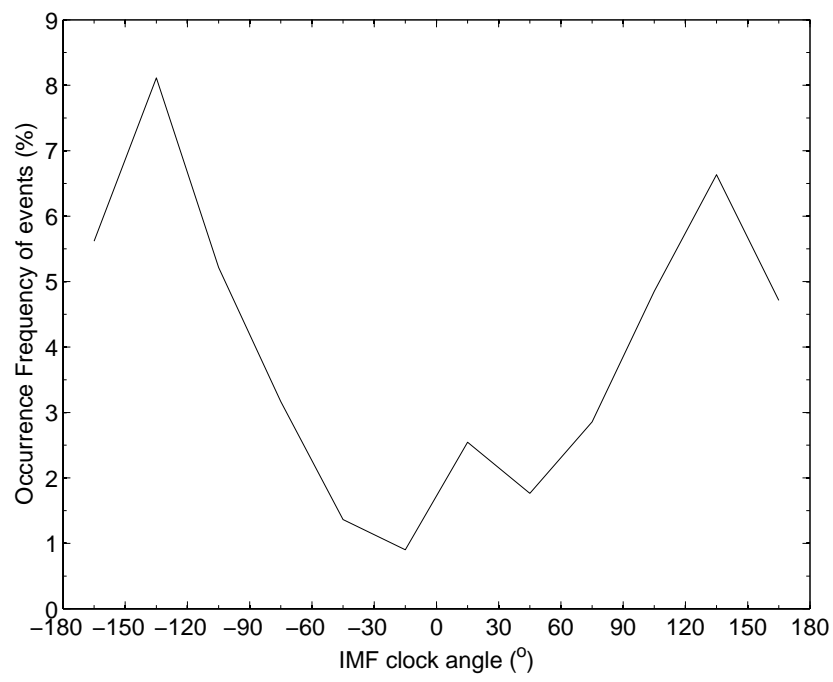

Fig. 4. Occurrence frequency of convection jets as a function of IMF clock angle.

ditions during which the jet events occur are therefore investigated. Figure 4 shows the occurrence frequency of jet events as a function of IMF clock angle, which is defined as $\theta=\tan ^{-1}\left(B_{\mathrm{y}} / B_{\mathrm{z}}\right)$ in GSM coordinates. The IMF and solar wind parameters have been propagated from ACE to the dayside magnetopause at $12 \mathrm{Re}$ by using all three velocity components of solar wind (The propagation has been done by the standard procedure described on the omni web page: http: //omniweb.gsfc.nasa.gov/html/HROdocum.html\#3). The solar wind and IMF parameters at the magnetopause are averaged over $20 \mathrm{~min}$ periods before the times of the event detection (Gérard et al., 2004). It can be seen that most of the events occur under southward IMF conditions, that is, $|\theta|>90^{\circ}$.

A superposed epoch analysis of the merging electric field, $\boldsymbol{E}_{\mathrm{m}}$ has been made. As the key time the detection of the flow burst was used. $\boldsymbol{E}_{\mathrm{m}}$ is defined as $v_{\mathrm{sw}} \sqrt{B_{\mathrm{y}}^{2}+B_{\mathrm{Z}}^{2}} \sin ^{2}(\theta / 2)$ (e.g. Kan and Lee, 1979), where $v_{\mathrm{sw}}$ is the solar wind velocity and $\theta$ is the clock angle of the IMF defined in GSM coordinates. Figure 5 shows the average evolution of $\boldsymbol{E}_{\mathrm{m}}$ one hour before and after the jets. It can be seen that $\boldsymbol{E}_{\mathrm{m}}$ shows an increase starting about $40 \mathrm{~min}$ before the jet. The peak value is reached at the time of the jet.

\subsection{Seasonal variation}

Figure 6 shows the occurrence frequency of jet events as a function of season. Most of the events occur in the winter season and least events occur during summer. This is as expected since the conductivity pattern is dominated by solar illumination in summer, which exhibits preferably large scale variations. While in winter, the conductivity is particle precipitation induced, generating more small-scale variations. 


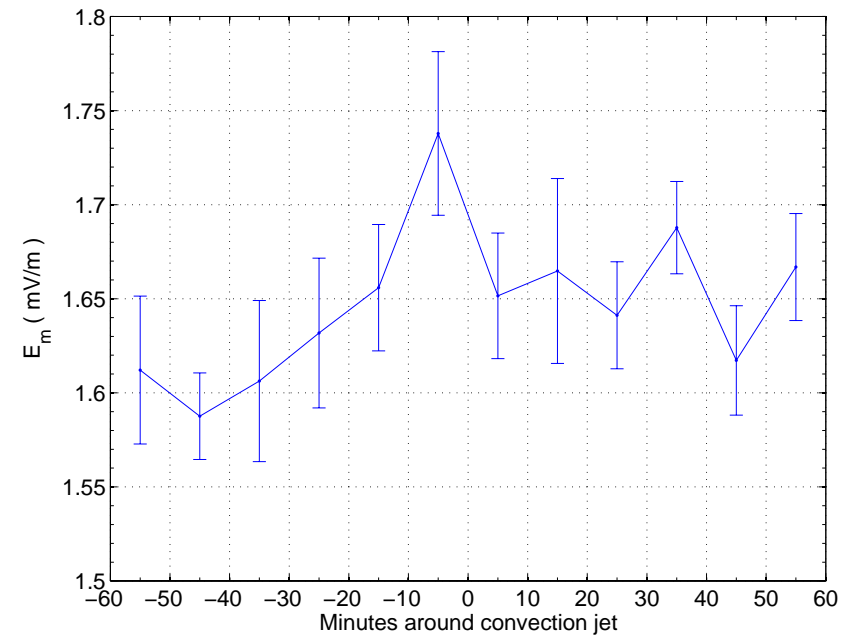

Fig. 5. Superposed epoch analysis of merging electric field, $\boldsymbol{E}_{\mathrm{m}}$, occurring during convection jets. The key time, 0 , corresponds to the jet observation time. $\boldsymbol{E}_{\mathrm{m}}$ has been propagated from the ACE satellite to the dayside magnetopause. The bars indicate the uncertainties of the 10 min averages.

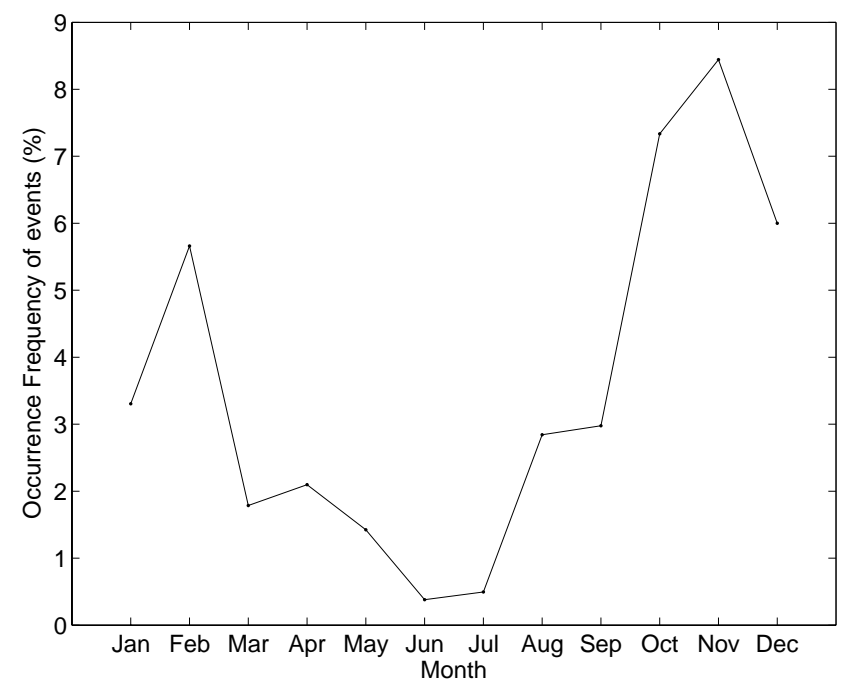

Fig. 6. Occurrence frequency of convection jet events as a function of season.

Figure 7 shows the velocity as a function of season (blue line). Overplotted is the monthly average of the conductivity gradient (green line). As expected, the Pedersen conductivity gradient is large in the winter and small in the summer. The velocity also maximizes in the winter and minimizes in the summer. The coincidence is clear for the months of June and December (see Fig. 7). But for all the other months no clear seasonal variation is emerging.

When we try to find a direct correspondence between the gradient of Pedersen conductivity and the jet velocity, we obtain a bad correlation between these two parameters, indicating there is no simple linear relation between these two quantities.

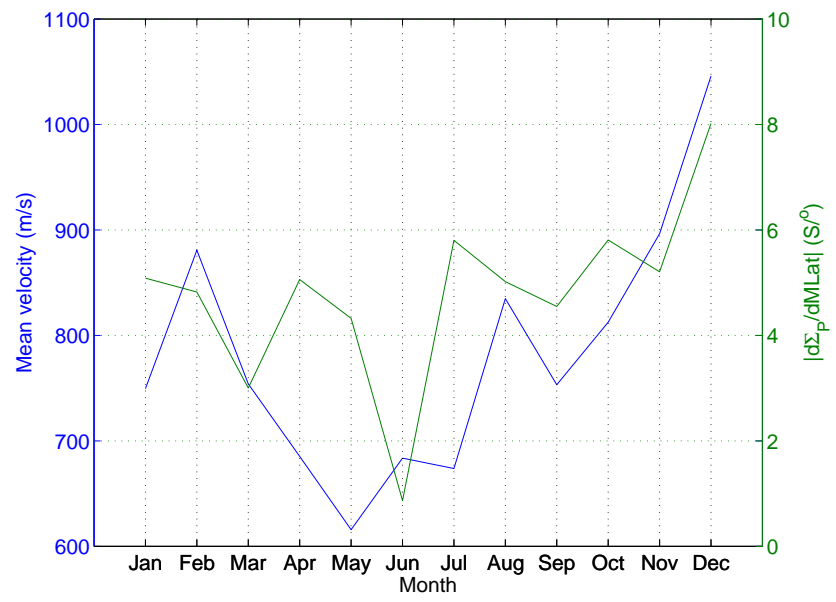

Fig. 7. Seasonal variation of the peak convection jet velocity value (blue) and $\left|d \Sigma_{\mathrm{P}} / d \mathrm{MLat}\right|$ (green). Monthly means are plotted.

\section{Discussion and conclusion}

In this work, we have shown that the cross-track velocity jets near the open-closed field line boundary can be associated with the peaks in ionospheric conductivity gradients. Both model and DMSP observations have been utilized to conduct this investigation. From the model test (Fig. 1) it can be seen, when the conductivity gradient is neglected in the calculation (that is, $\frac{\delta \Sigma_{\mathrm{P}}}{\delta \theta}=0$ ), the convection in the aurora region is reduced in magnitude with larger conductivity but the structure does not change too much at the poleward edge. For $\frac{\delta \Sigma_{\mathrm{P}}}{\delta \theta} \neq 0$, when the gradient of conductivity in the poleward boundary becomes sharper, there is a peak in $V_{\mathrm{y}}$ emerging near the poleward edge of the aurora (see Fig. 3 right frame), which resembles the flow bursts in DMSP observations. When one year of DMSP data are processed, hundreds of large ion flow events are identified with flow speeds larger than $500 \mathrm{~m} / \mathrm{s}$ occurring poleward of the aurora. Among them 280 (74\%) events are found to be associated with conductivity gradient peaks. The large probability of the coincidence between the jets and the conductivity gradients from observations as well as the model results may indicate that the conductivity gradient at the polar cap boundary is one important element in establishing the convection jets. The important effect of the conductivity gradient on the convection jets has also been noticed previously by Sandholt and Farrugia (2009). Based on several event studies at the dawn and dusk sectors, Sandholt and Farrugia (2009) found that for a small conductivity gradient the convection jet was absent, while in case of a sharp poleward boundary the fast $(2-3 \mathrm{~km} / \mathrm{s})$ flow can be observed.

When looking at the seasonal variation, it can be seen that most of the jet events occur in the winter season (Fig. 6), when conductivity gradients maximize (Fig. 7). Although we did not find a direct linear relationship between the jet velocity and the conductivity gradient, we observed that the 


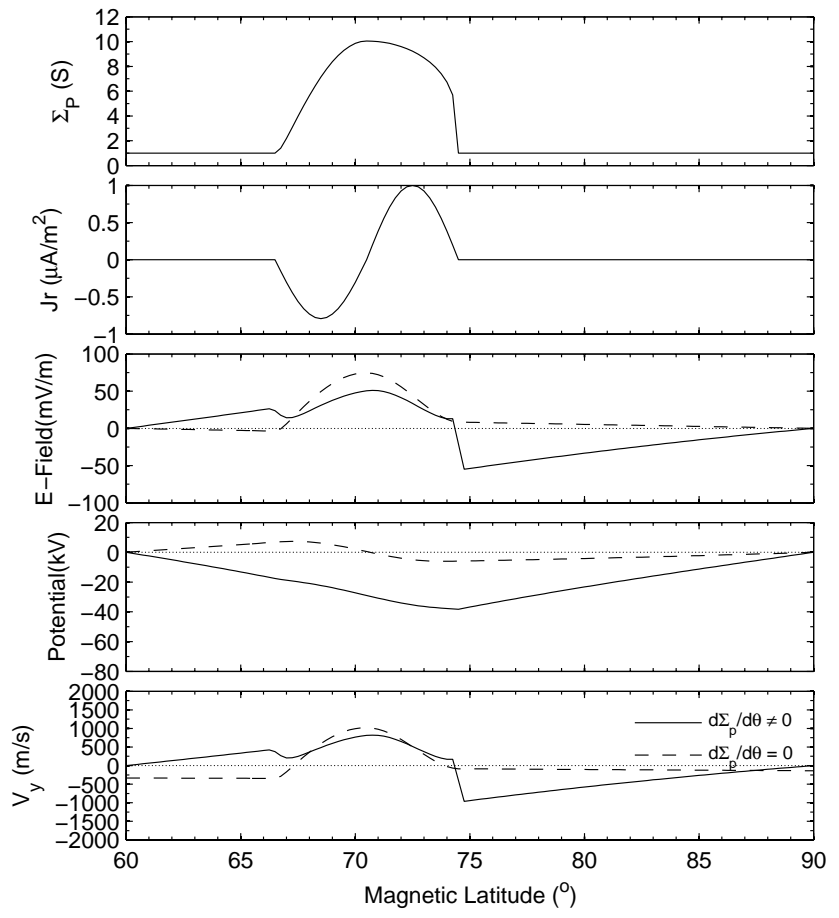

Fig. 8. Same as Fig. 1 except that Pedersen conductivity has a sin wave at the equatorward half and a $\sin ^{0.25}$ wave at the poleward half, which is a combination of the two conditions shown in Fig. 1. Field-aligned currents are kept unchanged.

convection jet maximizes in the winter season and minimizes in the summer season (Fig. 7).

The convection jets may be one element of the substorm processes (Hamza et al., 2000). We have checked the substorm onset event catalogue during 2002 as reported by Frey et al. (2004) and tried to find coincidences with convection peaks. Among the 378 rapid fast flow events, only 29 events occur less than $30 \mathrm{~min}$ after the onsets. We regard this as an indication that our convection jets are not related to substorms.

Supporting evidence for the way we explain the plasma flow jets at the poleward boundary comes from the superposed epoch analysis of the merging electric field, $\boldsymbol{E}_{\mathrm{m}}$. Figure 5 clearly reveals a rising $\boldsymbol{E}_{\mathrm{m}}$ during the $40 \mathrm{~min}$ before the appearing of the jets. It is known that for a larger $\boldsymbol{E}_{\mathrm{m}}$ the polar cap expands in size. This means, the temporal change of $\boldsymbol{E}_{\mathrm{m}}$ deduced from Fig. 5 implies an equatorward retreat of the auroral oval and the precipitation region. As a consequence the conductance poleward of the new oval position will decay rapidly. This forces the plasma to drift faster in order to maintain current continuity.

Sandholt and Farrugia (2009) found that the enhanced antisunward convection is mostly observed at the dusk side of the polar cap (18:00 MLT) during positive IMF $B_{\mathrm{y}}$ condition in the Northern Hemisphere. In our work the jet events in the 18:00-22:00 MLT sectors occur (see Fig. 4) with no IMF $B_{\mathrm{y}}$ polarity preference. The difference may be due to the different MLT ranges under investigation. The preferred occurrences of the jet events in the premidnight sector (see Fig. 3) makes sense. Here we expect the upward FACs on the poleward side of the oval, which are known to be more efficient in enhancing the conductivity. Also the latitude of highest event detections ( $\sim 72^{\circ}$ MLat) corresponds well with the latitude of R1 FAC for the average merging electric field of $\boldsymbol{E}_{\mathrm{m}}>1.6 \mathrm{mV} / \mathrm{m}$ prevailing before the event.

From a joint interpretation of observations and model predictions we propose a scenario for the generation of the plasma flow jet. Before the event we have a steady plasma convection. Due to magnetic field merging open flux is added to the tail and the polar cap expands. The equatorward retreat causes steep conductivity gradients to occur, especially on the duskside. On the equatorward edge the conductivity gradients are expected to be smooth after the polar cap expansion. For that reason plasma flow jet are forming at the poleward boundary of the premidnight auroral oval.

We have tried to reproduce this situation with our model. For the set up we used a combination of the two conditions shown in Fig. 1. The conductance profile has a sine wave shape in the lower latitude half and a $\sin ^{0.25}$ in the poleward half. Field-aligned currents are kept unchanged. The results are presented in Fig. 8. With these obvious features the observed plasma convection can be reproduced. There is a broad sunward plasma flow in the auroral region and the sudden switch to antisunward at the steep conductivity gradient.

In summary, we have provided convincing evidence that the steep conductivity gradients at the poleward boundary of the auroral oval in the premidnight sector are the cause for the plasma flow jets. These jets form preferably in the dark ionosphere where precipitating particles are the main cause for the conductivity. Flow jets do not seem to be related to substorm events, but they occur during times of enhanced reconnection when the polar cap expands.

Acknowledgements. We thank the Center for Space Sciences at the University of Texas at Dallas and the US Air Force for providing the DMSP IDM plasma data and the Space Physics Interactive Data Resource (SPIDR) for providing the DMSP SSJ/4 data. We thank ACE teams for providing solar wind and IMF data. This work is supported by National Nature Science Foundation of China (No. 40604017 and No. 40974096), open project founding of the State Key Laboratory of Space Weather of Chinese Academy of Sciences, and the Scientific Research Foundation for the Returned Overseas Chinese Scholars, State Education Ministry.

Topical Editor M. Pinnock thanks two anonymous referees for their help in evaluating this paper.

\section{References}

Amm, O.: Comment on "A three-dimensional, iterative mapping procedure for the implementation of an ionospheremagnetosphere anisotropic Ohm's law boundary condition in global magnetohydrodynamic simulations", Ann. Geophys., 14, 773-775, 1996, http://www.ann-geophys.net/14/773/1996/. 
Burke, W. J., Machuzak, J. S., Maynard, N. C., Basinska, E. M., Erickson, G. M., Hoffman, R. A., Slavin, J. A., and Hanson, W. B.: Auroral ionospheric signatures of the plasma sheet boundary layer in the evening sector, J. Geophys. Res., 99, 2489-2499, doi:10.1029/93JA02363, 1994.

Clauer, C. R. and Ridley, A. J.: Ionospheric observations of magnetospheric low-latitude boundary layer waves on August 4, 1991, J. Geophys. Res., 100, 21873-21884, doi:10.1029/95JA00678, 1995.

Fedder, J. A. and Lyon, J. G.: The solar wind-magnetosphereionosphere current-voltage relationship, J. Geophys. Res., 14, 880-883, 1987

Frey, H. U., Mende, S. B., Angelopoulos, V., and Donovan, E. F.: Substorm onset observations by IMAGE-FUV, J. Geophys. Res., 109, A10304, doi:10.1029/2004JA010607, 2004.

Gérard, J. C., Hubert, B., Grard, A., and Meurant, M.: Solar wind control of auroral substorm onset location observed with the IMAGE-FUV imagers, J. Geophys. Res., 109, A03208, doi:10.1029/2003JA010129, 2004.

Goodman, M. L.: A three-dimensional, iterative mapping procedure for the implementation of an ionosphere-magnetosphere anisotropic Ohm's law boundary condition in global magnetohydrodynamic simulations, Ann. Geophys., 13, 843-853, 1995, http://www.ann-geophys.net/13/843/1995/.

Grocott, A., Badman, S. V., Cowley, S. W. H., Yeoman, T. K., and Cripps, P. J.: The influence of IMF By on the nature of the nightside high-latitude ionospheric flow during intervals of positive IMF Bz, Ann. Geophys., 22, 1755-1764, 2004, http://www.ann-geophys.net/22/1755/2004/.

Grocott, A., Milan, S. E., and Yeoman, T. K.: Interplanetary magnetic field control of fast azimuthal flows in the nightside high-latitude ionosphere, Geophys. Res. Lett., 35, L08102, doi:10.1029/2008GL033 545, 2008.

Hamza, A. M., Huber, M., Lyatsky, W., Kustov, A. V., Andre, D., and Sofko, G.: Eastward convection jet at the poleward boundary of the nightside auroral oval, Geophys. Res. Lett., 27, 28092812, doi:10.1029/2000GL003745, 2000.

Hardy, D. A., Schmitt, L. K., Gussenhoven, M. S., Marshall, F. J., and Yeh, H. C.: Precipitating electron and ion detectors (SSJ/4) for the block 5D/Flights 6-10 DMSP (Defense Meteorological Satellite Program) satellites: Calibration and data presentation, Rep. AFGL-TR-84-0314, Air Force Geophys. Lab., Air Force Base, MA, 1984.

Hardy, D. A., Gussenhoven, M. S., Raistrick, R., and Mcneil, W. J.: Statistical and functional representations of the pattern of auroral energy flux, number flux, and conductivity, J. Geophys. Res., 92, 12275-12294, 1987.

Heppner, J. P. and Maynard, N. C.: Empirical high-latitude electric field models, J. Geophys. Res., 92, 4467-4489, 1987.

Kan, J. R. and Lee, L. C.: Energy coupling function and solar windmagnetosphere dynamo, Geophys. Res. Lett., 6, 577-580, 1979.

Karlsson, T. and Marklund, G. T.: A statistical study of intense lowaltitude electric fields observed by Freja, Geophys. Res. Lett., 23, 1005-1008, doi:10.1029/96GL00773, 1996.

Lyons, L. R., Lu, G., de la Beaujardière, O., and Rich, F. J.: Synoptic maps of polar caps for stable interplanetary magnetic field intervals during January 1992 geospace environment modeling campaign, J. Geophys. Res., 101, 27283-27298, doi:10.1029/ 96JA02457, 1996.
Milan, S. E., Baddeley, L. J., Lester, M., and Sato, N.: A seasonal variation in the convection response to IMF orientation, Geophys. Res. Lett., 28, 471-474, doi:10.1029/2000GL012245, 2001.

Provan, G. and Yeoman, T. K.: Statistical observations of the MLT, latitude and size of pulsed ionospheric flows with the CUTLASS Finland radar, Ann. Geophys., 17, 855-867, 1999 . http://www.ann-geophys.net/17/855/1999/.

Raeder, J., McPherron, R. L., Frank, L. A., Kokubun, S., Lu, G., Mukai, T., Paterson, W. R., Sigwarth, J. B., Singer, H. J., and Slavin, J. A.: Global simulation of the Geospace Environment Modeling substorm challenge event, J. Geophys. Res., 106, 381396, 2001.

Rich, F. J. and Hairston, M.: Large-scale convection patterns observed by DMSP, J. Geophys. Res., 99, 3827-3844, 1994.

Richmond, A. D. and Kamide, Y.: Mapping electrodynamic features of the high-latitude ionosphere from localized observations - Technique, J. Geophys. Res., 93, 5741-5759, doi:10.1029/ JA093iA06p05741, 1988.

Ridley, A. J. and Clauer, C. R.: Characterization of the dynamic variations of the dayside high-latitude ionospheric convection reversal boundary and relationship to interplanetary magnetic field orientation, J. Geophys. Res., 101, 10919-10938, doi: 10.1029/95JA03805, 1996

Ridley, A. J., Hansen, K. C., Tóth, G., Zeeuw, D. L. D., Gombosi, T. I., and Powell, K. G.: University of Michigan MHD results of the GGCM Metrics challenge, J. Geophys. Res., 107(A10), 1290, doi:10.1029/2001JA000253, 2002.

Ridley, A. J., Gombosi, T. I., and DeZeeuw, D. L.: Ionospheric control of the magnetosphere: conductance, Ann. Geophys., 22 567-584, 2004, http://www.ann-geophys.net/22/567/2004/.

Robinson, R. M., Vondrak, R. R., Miller, K., Dabbs, T., and Hardy, D.: On calculating ionospheric conductances from the flux and energy of precipitating electrons, J. Geophys. Res., J. Geophys. Res., 92, 2565-2569, 1987.

Ruohoniemi, J. M. and Greenwald, R. A.: Statistical patterns of the high-latitude convection obtained from Goose Bay HF radar observations, J. Geophys. Res., 101, 21743-21763, 1996.

Ruohoniemi, J. M. and Greenwald, R. A.: Dependencies of high-latitude plasma convection: Consideration of interplanetary magnetic field, seasonal, and universal time factors in statistical patterns, J. Geophys. Res., 110, A09204, doi:10.1029/ 2004JA010815, 2005.

Sandholt, P. E. and Farrugia, C. J.: Plasma flow channels at the dawn/dusk polar cap boundaries: momentum transfer on old open field lines and the roles of IMF $B_{y}$ and conductivity gradients, Ann. Geophys., 27, 1527-1554, 2009, http://www.ann-geophys.net/27/1527/2009/.

Troshichev, O. A., Shishkina, E. M., Meng, C., and Newell, P. T.: Identification of the poleward boundary of the auroral oval using characteristics of ion precipitation, J. Geophys. Res., 101, 50355046, doi:10.1029/95JA03634, 1996.

Wang, H., Ridley, A. J., and Lühr, H.: Validation of the Space Weather Modeling Framework using observations from CHAMP and DMSP, Space Weather, 6, S03001, doi:10.1029/ 2007SW000355, 2008

Weimer, D. R.: A flexible, IMF dependent model of high-latitude electric potential having "space weather" applications, Geophys. Res. Lett., 23, 2549-2552, 1996. 